\title{
Stabilization of some composite stochastic control systems with nontrivial solutions
}

\begin{abstract}
This paper deals with the global stability for some composite stochastic control systems with nontrivial solutions by means of dynamic feedback laws. In particular, we establish feedback law for global asymptotic stabilization of a control subsystem deduced from the composite stochastic system and apply the result to stabilize the original composite stochastic system. Under this framework, firstly we derive necessary and sufficient conditions for the existence of feedback law which renders a ball that contains all nontrivial equilibrium solutions of the stochastic control subsystem globally asymptotically stable in probability. Secondly, for the composite system, we give the sufficient conditions for practical global asymptotic stability of the composite system based upon the feedback law derived for the subsystem. Numerical examples are given to validate our results.
\end{abstract}

Keyword: Stochastic control system; Composite system; Control Lyapunov function; Practical global asymptotic stability in probability; Nontrivial solutions 
